\title{
Development of Human Postural Balance Monitoring System in Bipedal Standing
}

\author{
Yakub Fahim Luckyarno, Sunarno \\ Department of Engineering Physics, Faculty of Engineering, \\ Universitas Gadjah Mada \\ Jalan Grafika 2, Bulaksumur, Yogyakarta 55281, Indonesia \\ E-mail: sunarno@ugm.ac.id \\ Rony Wijaya \\ Unit of Production House, Faculty of Medicine, \\ Universitas Gadjah Mada \\ Jalan Medika Sekip Utara, Bulaksumur, Yogyakarta 55281, Indonesia \\ E-mail: rony_wijaya@yahoo.com
}

\begin{abstract}
A monitoring of human postural balance in bipedal standing is an important parameter in medicine, related to balance disorder. A Human Postural Balance Monitoring System in Bipedal Standing (HPBMSBS) consists of a 3axis digital position sensor module, a Programmable System-on-Chip (PSoC) 4, and a PC-display. It can display directions of postural balance in 8 positions, Forward, Backward, Left, Right, Right-Forward, Left-Forward, RightBackward, Left-Backward. Details related to the system will be explained in this paper.
\end{abstract}

Keywords: Balance disorder, position sensor, PSoC, PC-display.

\section{Introduction}

Human balance in bipedal standing becomes an important parameter in medicine. Nowadays, information about human balance is used by doctors in order to do a higher-level research. A research about human body balance is conducted by Faculty of Medicine, Universitas Gadjah Mada. It needs an instrument to know the biggest probability of human body sway.

Definition about bipedal standing or standing position is explained by ${ }^{1}$. A human body in bipedal standing position is a superposition between head, body and legs, in a longitudinal axis. If one or more parts does not meet the requirement or condition, then a superposition will not be happened, so human cannot stand upright.

Former research about Human Postural Balance Monitoring System in Bipedal Standing (HPBMSBS) had been conducted. Generally, research by ${ }^{2,3}$ have a same basic system design, consists of sensor, processor, and display. The difference is on what kind of sensor used to. Four load cell sensors are used by ${ }^{2}$, meanwhile three strain gage sensors are used by ${ }^{3}$. Another research is conducted by ${ }^{4}$, in which uses an accelerometer sensor module as a position sensor. An HPBMSBS built by Production House Unit, Faculty of Medicine, Universitas Gadjah Mada has been developed for several times, especially on development of sensor. The latest development of HPBMSBS is using two position sensor modules. In this paper, will explain development of HPBMSBS from former research and former instrument, using a position sensor module and different processor.

\section{Method of Research}

Method of research is shown by Fig. 1. 


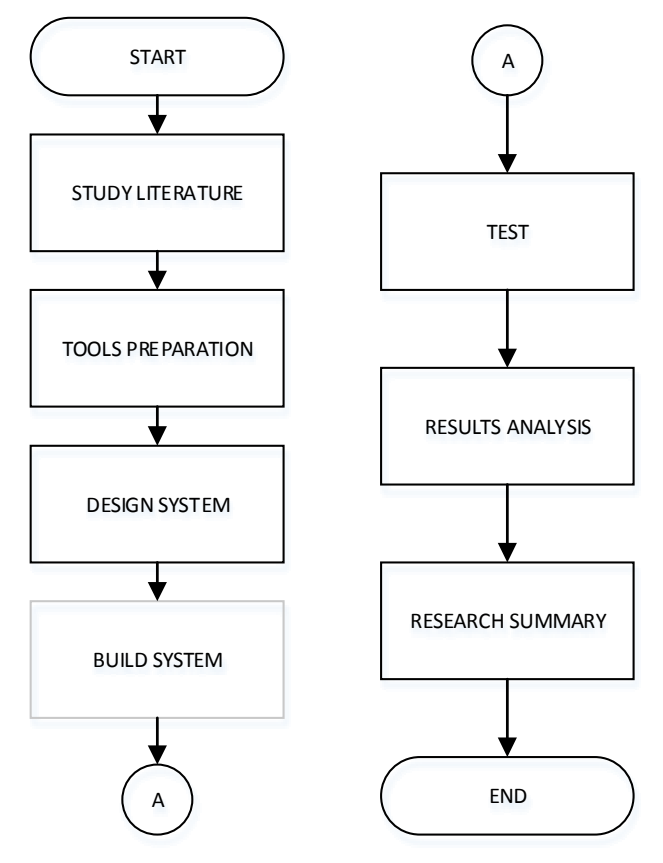

Fig. 1. Method of research

\subsection{Sensor System}

A sensor system is a system consists of a platform and a position sensor module. Platform is made from iron plate, a $41-\mathrm{cm}$ in diameter. Four springs are mounted between upper and lower plate, have function to maintain the platform's position in starting position.

This system uses a position sensor module, utilizing a 3axis accelerometer sensor module. Sensor capture the phenomenon of distance difference between a static plate and a dynamic plate, and convert it into a digital value. A diagram block of sensor shown by Fig. 2 .

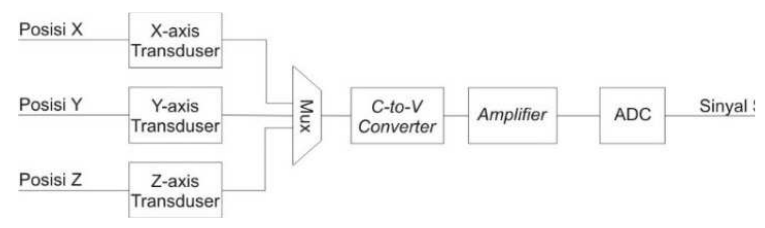

Fig. 2. Diagram Block of Sensor

\subsection{Processor}

A Programmable System-on-Chip (PSoC) 4 is used. Its function is to read data from sensor module and send data to display in PC. It uses two kinds of serial communication, I2C and UART. This processor is higher in specification than processor in ${ }^{2-4}$. It has 32-bit ARM Cortex-M0, UART baudrate up to 115200 , clock up to $64 \mathrm{MHz}$, and programmable digital and analog block. Based on the architecture and function, $\mathrm{PSoC}$ is placed between ordinary microcontroller and Field Programmable Gate Array (FPGA).

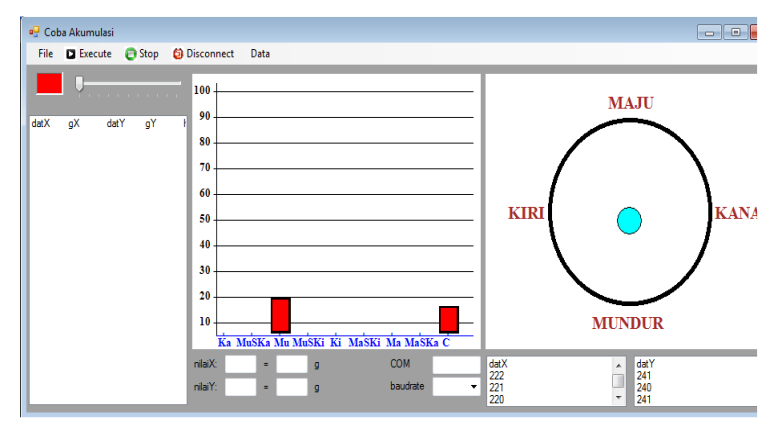

\subsection{Display}

A display in $\mathrm{PC}$ is used to give information about the way the body sway. It looks like in Fig. 3.

Fig. 3. Display in PC/laptop

\subsection{Test}

Several tests are performed to determine the characteristics of the system. The tests are sensor test, interface test, and system test.

\section{Result and Analysis}

This chapter contains result and analysis of sensor test, interface test, and system test.

\subsection{Sensor Test}

The purpose of sensor test is to know how to get data from sensor module and get its characteristics. Sensor is tested by giving input, which is a force, to the sensor's direction and observe the output. Directions given to the sensor are $\mathrm{X}+, \mathrm{Y}+, \mathrm{X}-$, and $\mathrm{Y}-$ (shown in Fig. 4). The desired output is a value in each direction.

The test result is shown by Fig. 5 . 


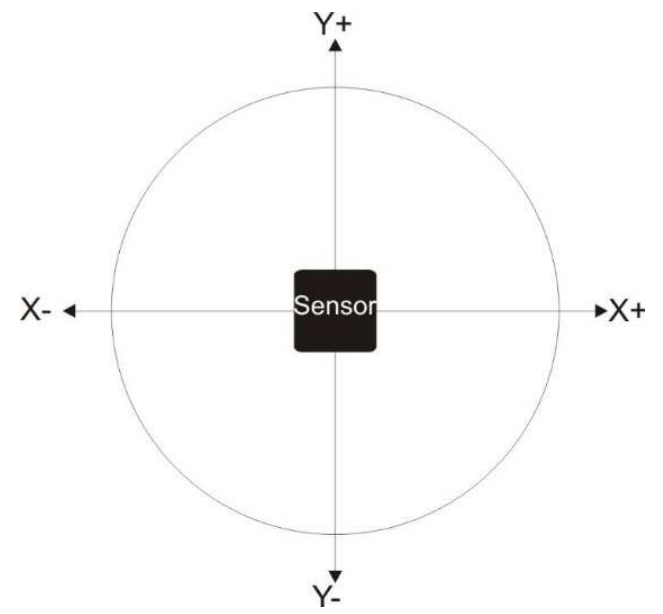

Fig. 4. Direction of Sensor Test

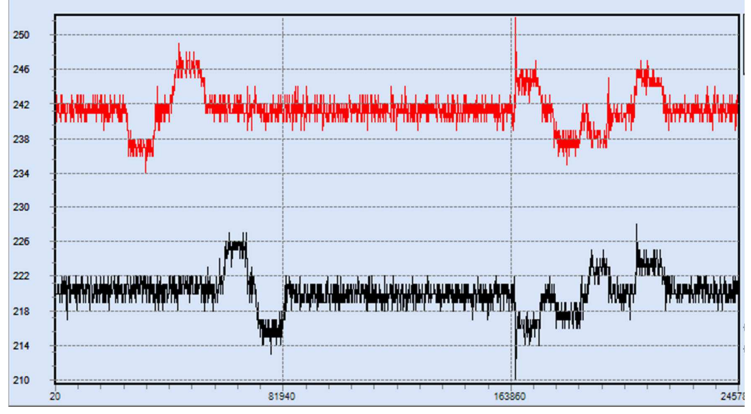

Fig. 5. Result of Sensor Test

The red line in Fig. 5 is test result for $\mathrm{X}$ direction and black line is test result for $\mathrm{Y}$ direction. A range of value from 240 to 243 shows $\mathrm{X}$ direction in a steady position, means there is no force in that direction. Values more than 243 show the $\mathrm{X}$ - direction and values less than 240 show the $\mathrm{X}+$ direction. A range of value from 219 to 221 shows that $\mathrm{Y}$ direction in a steady position. Values more than 221 show $\mathrm{Y}$ - direction and values less than 219 show $\mathrm{Y}+$ direction.

\subsection{Communication Test}

Communication test is performed to make sure that there is no error when data communication process is occurred. Parameter that varied is baudrate. Variations of baudrate value are 1200, 2400, 4800, 9600, 19200, 38400,57600 , and 115200 . Result of test is shown by Fig. 6 and Fig. 7.

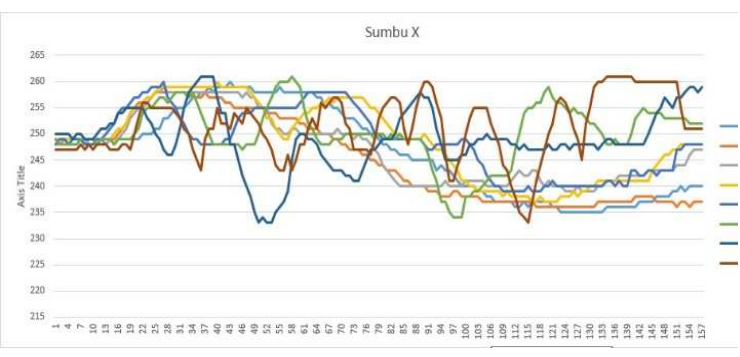

Fig. 6. Data X Direction

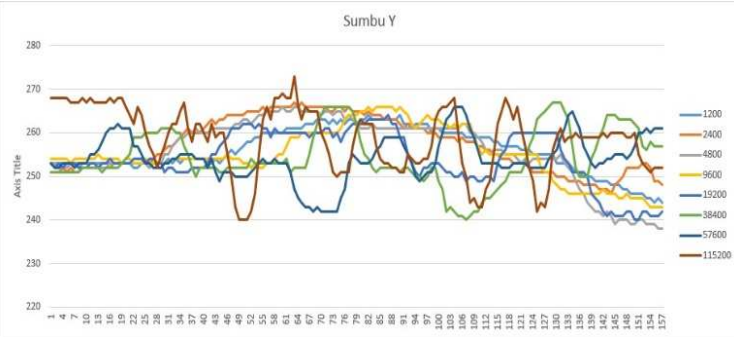

Fig. 7. Data Y Direction

As shown by Fig. 6 and Fig. 7, process of data communication is doing well. Some baudrate values show the steadiness of value and others show fluctuations of value. It happens because data from sensor is given randomly in direction. Even some values show fluctuation, they still can be used for data communication, because delay in the process is 125 millisecond.

\subsection{System Test}

System test is performed to know the ability of reading data from sensor and is displayed in PC. The test result is shown in Table. 1.

Table. 1. Display Test Result

\begin{tabular}{|l|l|}
\hline Codel & Direction \\
\hline 0 & Center \\
\hline 1 & Right-Forward \\
\hline 2 & Right-Backward \\
\hline 3 & Left-Forward \\
\hline 4 & Left-Backward \\
\hline 5 & Forward \\
\hline 6 & Backward \\
\hline 7 & Right \\
\hline 8 & Left \\
\hline
\end{tabular}


Table. 1 shows codes used in display. Codes contain certain range of value from sensor, as shown by Table. 2.

Table. 2. Result of Data Processing

\begin{tabular}{|c|c|c|}
\hline NilaiX & NilaiY & Code \\
\hline $240<$ NilaiX $<243$ & $219<$ NilaiY $<221$ & 0 \\
\hline NilaiX $<240$ & NilaiY $<219$ & 1 \\
\hline NilaiX $<240$ & NilaiY $>221$ & 2 \\
\hline NilaiX $>243$ & NilaiY <219 & 3 \\
\hline NilaiX $>243$ & NilaiY $>221$ & 4 \\
\hline $240<$ NilaiX < 243 & NilaiY <219 & 5 \\
\hline $240<$ NilaiX < 243 & NilaiY $>221$ & 6 \\
\hline NilaiX $<240$ & $219<$ NilaiY < 221 & 7 \\
\hline NilaiX $>243$ & $219<$ NilaiY <221 & 8 \\
\hline
\end{tabular}

NilaiX and NilaiY are variable presenting value of sensor in each $\mathrm{X}$ direction and $\mathrm{Y}$ direction.

\section{Discussion}

Based on the result, the HPBMSBS can read data from sensor in $\mathrm{X}$ and $\mathrm{Y}$ direction. Differences from ${ }^{2}$ and ${ }^{3}$ are in the use of sensor and how to give information about body sway using display. Result of this paper is really useful for doctors, especially in anatomy and analysis of posture. This instrument needs more improvement and reliability test, so it can be used widely and safely.

\section{Acknowledgements}

This research was technically supported by Laboratory of Sensor and Telecontrolling System, Department of Engineering Physics, Faculty of Engineering, Universitas Gadjah Mada and financially supported by Unit of Production House, Faculty of Medicine, Universitas Gadjah Mada.

\section{References}

1. A.M. Bronstein, T. Brandt, M.H. Woollacott, J.G. Nutt, Clinical Disorders of Balance, Posture, and Gait (Arnold, London, 2014).

2. M. Gohari, N. Mortaza, Designing and Fabrication of a Postural Stabilometer: A Static Platform, in Proceedings of the $17^{\text {th }}$ Iranian Conference of Biomedical Engineering, (Isfahan, Iran, 2010), pp. 1-4.

3. S. Boukhenous, M. Attari, Y. Remram, Force Platform for Postural Balance Analysis, in The $11^{\text {th }}$ International
Conference on Information Sciences, Signal Processing and their Applications, (Montreal, QC, Canada, 2012), pp. 854-858.

4. A.D. Caballero, J.J.C. Lopez, An Accelerometer-based Embedded System-on-Chip for Measuring Human Body Joint Angles, in 2013 Pan American Health Care Exchanges (PAHCE), (Medellin, Colombia, 2013), pp. 1. 